\title{
COMPARATIVE STUDY BETWEEN OUTCOMES OF EARLY PRIMARY ENDOSCOPIC REALIGNMENT VERSUS SUPRAPUBIC CYSTOSTOMY WITH DELAYED RECONSTRUCTION IN THE MANAGEMENT OF POSTERIOR URETHRAL INJURY
}

\author{
MRINMOY BISWAS ${ }^{1}$ MD.FAZAL NASER ${ }^{2}$, SM MAHBUB ALAM $^{3}$, MD. ABUL HOSSAIN², M A AWAL ${ }^{4}$ \\ ${ }^{1}$ Department of Urology, Sir Salimullah Medical College \& Mitford Hospital, Dhaka, ${ }^{2}$ Department of Urology, Shaheed \\ Suhrawardy Medical College, Dhaka, ${ }^{3}$ Department of Urology, Dhaka Medical College, Dhaka, ${ }^{4}$ Department of Urology, \\ Sher-e-Bangla Medical College, Barisal
}

\begin{abstract}
:
Objectives: To compare the outcomes of early primary endoscopic realignment and suprapubic cystostomy with the outcomes of delayed reconstruction in the management of posterior urethral injury.

Methods: This is a quasi-experimental prospective study Conducted in the Urology department Dhaka medical college and hospital from January 2009 to December 2010. Atotal of 60 consecutive patients were selected for this study and inclusion criteria, male patients and age 18years and above. Posterior urethral injury resulting in distraction defect and urethral injury with pelvic fracture. Group-A, 28 patients underwent early primary endoscopic realignment after initial suprapubic urinary diversion and Group-B, 32 patients underwent primary suprapubic urinary diversion and delayed urethral reconstruction after 3 months of injury.

Results: After procedure 21(75\%) out of 28 patients in Group-A developed stricture 12(42.9\%) had simple and short segment stricture and 7(32.1\%) had simple and long segment stricture, while in Group-B 12(37.5\%) patients developed simple and short segment stricture. All of the patients in Group-A had minimum blood loss during operation. While, majority (81.3\%) of patients in Group-B had a history of maximum blood loss. None of the patients in Group-A developed incontinence throughout the observations period (from removal of catheter to 9 months), while $15.6 \%$ of the patients in Group-B had incontinence at removal of catheter after anastomotic urethroplasty and at 3 month. The incontinence further increased to $18.8 \%$ at 6 and 9 months. Complaints of impotence were significantly less in Group-A than that in Group-B throughout the period of observation (14.3\% vs. $37.5 \%, p=0.042 ; 14.3 \%$ vs. $37.5 \%, p=0.042$ and $14.3 \%$ vs. $37.5 \%, p=0.042)$. At removal of urethral catheter, stricture formation was observed and statistically there was no difference in Group-A and Group-B (14.3\% vs. $21.9 \%$ in Group-B, $p=0.448)$. At month 3 , stricture formation was significantly higher in Group-A than that in Group-B (42.9\% vs. $15.6 \%, p=0.020)$. At month 6 , about $18 \%$ of patients in Group-A had stricture, but none of patients in Group-B was found so $(p=0.018)$.

Conclusions: Early primary endoscopic realignment of traumatic posterior urethral disruption is a simple, less traumatic, safe, and rapid technique. It provides a low morbidity and less postoperative complications. Though recurrent stricture formation is higher but the strictures are simple and short and amenable to be corrected endoscopically. It may be considered as initial therapy in the management of posterior urethral distraction defect over suprapubic cystostomy with delayed reconstruction.
\end{abstract}

Keywords: Urethra, endoscopic realignment, suprapubic cystostomy, stricture.

Bangladesh J. Urol. 2014; 17(1): 29-35

Correspondences: Mrinmoy Biswas, Department of Urology, Sir Salimullah Medical College \& Mitford Hospital, Dhaka. Email: dr_mrinalroy@yahoo.com 


\section{Introduction:}

Urethral injuries are uncommon and occur most often in men, usually associated with pelvic fractures or falling astride injury, but rare in women. The management of posterior urethral disruption secondary to blunt pelvic injury several authors have reported encouraging results with endoscopic realignment as primary therapy. This procedure requires immediate suprapubic diversion followed by delayed or early endoscopic realignment and is associated with decreased intra-operative blood loss, shortened operative time, decreased length of hospital stay, and less incontinence than open urethroplasty. In conventional classical approach is early suprapubic cystostomy with delayed urethral reconstruction, virtually $100 \%$ of patients treated with a suprapubic tube (catheter) and delayed reconstruction require urethroplasty, while early realignment obviate the need for about half of these operations[1].

Urethral injuries are uncommon and occur most often in men, usually associated with pelvic fractures or falling astride injury, but rare in women. Various parts of the urethra may be lacerated, transected, or contused; but the post-traumatic rupture of the posterior urethra occurs in $10-25 \%$ of patients with pelvic fractures. These lesions are secondary to the highway accidents in $75 \%$ of cases[1,2]. Because the posterior urethra is fixed at both the urogenital diaphragm and the puboprostatic ligaments, the bulbomembranous junction is more vulnerable to injury during pelvic fracture[3]. The injuries vary from simple stretching (25\%) to partial rupture $(25 \%$ ) to complete disruption (50\%). And the posterior urethral disruption injuries typically occur in conjunction with multi-system trauma from vehicular accident, fall, or industrial accidents resulting in pubic diastasis, localized pubic rami fractures, or more complex pelvic fractures[4]. Though posterior urethral injuries are not common; these injuries are associated with considerable morbidity, including urinary incontinence, erectile dysfunction and urethral strictures. These complications can lead to chronic disability, resulting in long-term physical and emotional distress as well as significant financial loss and some of these complications may be increased in magnitude or newly produced after getting definitive treatment[1,5]. As for example, the immediate open surgical manipulation of the injured site to achieve realignment or suturing of the disrupted urethra may cause more damage to the sphincteric mechanism or to the nerve concerned and produce aggravated form of incontinence or impotence[6].

Therefore, it is very much important to evaluate the patient properly and to select the immediate and early management as well as delayed treatment modalities. Here, we are concerned with the management of posterior urethral injuries specially, complete disruption injury associated with pelvic fracture.

The management of posterior urethral disruption secondary to blunt pelvic injury remains controversial ${ }^{5}$. In the past urethral disruption was treated with open primary realignment or suprapubic diversion with delayed urethroplasty. Delayed open urethroplasty, as described by Waterhouse and others, was considered the gold standard repair[7]. Immediate open realignment was difficult due to tissue trauma and poor visibility associated with pelvic bleeding. In addition, the incidence of impotence and incontinence associated with open realignment was significantly higher than with delayed urethroplasty[5].

Recently, several authors have reported encouraging results with endoscopic realignment as primary therapy. This procedure requires immediate suprapubic diversion followed by delayed or early endoscopic realignment and is associated with decreased intra-operative blood loss, shortened operative time, decreased length of hospital stay, and less incontinence than open urethroplasty[7].Some experts have reported that this technique is unhelpful, but they are small in number ${ }^{8}$. In conventional classical approach i.e. early suprapubic cystostomy with delayed urethral reconstruction, virtually $100 \%$ of patients treated with a suprapubic tube (catheter) and delayed reconstruction require urethroplasty, while early realignment obviate the need for about half of these operations[8]. On the other hand, series that advocate primary realignment show a lower rate of urethroplasty as well as the formation of potentially less significant strictures than those treated primary cystostomy alone. They may be more amenable to endoscopic core through treatment, which often fail in the longer strictures seen with delayed therapy. They also believe that subsequent open repair, if needed, simpler than that in the delayed urethroplasty also conducted a prospective study on 21 patients having urethral injury in one center[9]. They compared between outcomes of immediate endoscopic realignment (IER) versus delayed urethral reconstruction. They concluded that IER results in a significantly reduced time to spontaneous voiding with less risk of urethral stricture.

And very recently, conducted an analytical observation by collecting data from studies during last decades regarding the management of urethral injuries[10]. They also concluded that primary urethral realignment (either 
surgical with minimal paravesical dissection or endoscopical) should be preferred option for the initial management of posterior urethral injuries.

Therefore, keeping in mind these benefits, here we have performed this study.

\section{Materials and Methods:}

This is a quasi-experimental prospective study Conducted in the Urology department Dhaka medical college and hospital from January 2009 to December 2010.

Atotal of 60 consecutive patients were selected for this study and inclusion criteria, male patients and age 18years and above. Posterior urethral injury resulting in distraction defect and urethral injury with pelvic fracture. Group-A, 28 patients underwent early primary endoscopic realignment after initial suprapubic urinary diversion and Group-B, 32 patients underwent primary suprapubic urinary diversion and delayed urethral reconstruction after 3 months of injury.

Per-operative blood loss: minimum/maximum, Operation time: less time consuming/more time consuming, Postoperative hospital stay: minimum/maximum, Length of the stricture: short/long; short means 1 or less than 1 $\mathrm{cm}$, Nature of the stricture: simple/complex; complex means associated with fistula, diverticulum, very long segment stricture i.e. longer than $3 \mathrm{~cm}$, , Procedures applied to correct strictures: simple optical internal urethrotomy or anastomotic urethroplasty with or without partial or total pubectomy, Operative complications: incontinence, impotence, and secondary stricture.

After diagnosis of urethral injury the initial urologic management was suprapubic urinary diversion by suprapubic catheter insertion. Then all the patients were counseled accordingly to be included in the study groups. After getting consent the patients were allocated into two groups through purposive sampling technique; Group A and Group B.

Group A-All the patients here, underwent early primary endoscopic realignment after initial suprapubic urinary diversion and follow up. Group B- all the patients were treated by primary suprapubic urinary diversion through suprapubic catheter insertion and delayed urethral reconstruction after 3 months.

Group A: A cystogram through the suprapubic catheter, evaluated bladder neck competence and the length of the posterior urethra. This procedure was combined with a retrograde urethrogram to determine the length of the defect under antibiotic coverage. In some instances, C.T. scan of abdomen and pelvis was done to complete the evaluation of the urinary tract injury. The timing of urethral realignment was determined by the associated injuries. If there was an isolated urethral injury, realignment was performed as soon as the condition was stable. Urethral realignment was delayed in case of hemodynamic instability or life threatening injuries that precluded urological manipulation. The time period ranged from 7 to 14 days.

\section{Observation and Results:}

The mean age of the patients was almost similar between the groups ( $28.8 \pm 8.4$ vs. $27.4 \pm 7.2$ years, $p=0.486$ ). Genito-urinary examination depicts that all of the patientsinGroup-Aand Group-B exhibited blood at meatus, pelvic fracture and suprapubic fullness and tenderness. Over two-third (67.9\%) of patients in Group$A$ and $71.9 \%$ in Group-B had perineal haematoma. Before procedure 7 (21.9\%) patients in Group-B developed simple and long segmented stricture and 25(78.1\%) patients exhibited complex stricture. After procedure $21(75 \%)$ out of 28 patients in Group-A developed stricture $12(42.9 \%)$ had simple and short segment stricture and $7(32.1 \%)$ had simple and long segment stricture, while in Group-B 12(37.5\%) patients developed simple and short segment stricture. All of the patients in Group-A had minimum blood loss during operation. While, majority (81.3\%) of patients in Group$B$ had a history of maximum blood loss. All of the patients in Group-A required operation time 2 hours or less than 2 hours. Majority (87.5\%) of patients in Group-B needed for operation more than 2 hours and $12.5 \% 2$ hours or less than 2 hours. The mean duration of operation was found significantly higher in Group-B than that in Group$\mathrm{A}(2.5 \pm 0.4$ vs. $1.2 \pm 0.4 \mathrm{hrs}, \mathrm{p}<0.001)$. All the patients in Group-A had duration of hospital stay 3 days or less than 3 days at the same time all patients in Group-B had more than 3 days after operation. The mean hospital stay was about two times higher in Group-B compared to Group-A ( $6.9 \pm 1.8$ vs. $3.0 \pm 0.1$ days, $p<0.001)$. None of the patients in Group-A developed incontinence throughout the observations period (from removal of catheter to 9 months), while $15.6 \%$ of the patients in Group-B had incontinence at removal of catheter after anastomotic urethroplasty and at 3 month. The incontinence further increased to $18.8 \%$ at 6 and 9 months. Complaints of impotence were significantly less in Group-A than that in Group-B throughout the period of observation ( $14.3 \%$ vs. $37.5 \%, p=0.042 ; 14.3 \%$ vs. 
$37.5 \%, p=0.042$ and $14.3 \%$ vs. $37.5 \%, p=0.042)$. At removal of urethral catheter, stricture formation was observed and statistically there was no difference in Group-A and Group-B (14.3\% vs. $21.9 \%$ in Group-B, p $=0.448)$. At month 3 , stricture formation was significantly higher in Group-A than that in Group-B (42.9\% vs. $15.6 \%, p=0.020$ ). At month 6 , about $18 \%$ of patients in Group-A had stricture, but none of patients in Group-B was found so $(p=0.018)$.

\section{Discussion:}

Urethral injuries are uncommon and occur most often in men, usually associated with pelvic fractures or falling astride injuries, but rare in women. Various parts of the urethra may be lacerated, transected, or contused, but the post-traumatic rupture of posterior urethra occurs in $10-25 \%$ of patients with pelvic fractures. These lesions are secondary to the highway accidents in $75 \%$ of cases[1,2].

Of all injuries to the entire urinary tract the most serious is that which affects the posterior urethra, not only because of its location deep in the pelvis but mainly because of the sphincter active urethra as well as the intimate relationship to the nervi erigentes. In every pelvic fracture urethral injury, there is the potential risk of 3 complications, that is stricture, incontinence, and impotence. These complications may result directly from original trauma and/or iatrogenic trauma induced by the immediate treatment selected[2].

The management of posterior urethral disruption secondary to blunt pelvic injury remains controversial[5,12]. It represents a challenge to the urologist[13]. The goals of treatment of posterior urethral injury should be a patent continent urethra and maintenance of sexual potency as before trauma. To achieve these goals several methods have been used, including primary suturing of the distracted urethral ends, primary realignment with urethral splinting (with or without traction and performed surgically or endoscopically) and suprapubic cystostomy with delayed repair[11].

Immediate end to end anastomosis or surgical exploration with realignment over a catheter exposes the severely injured patient to further risks. The operation can be complicated by considerable haemorrhage and/ or by difficulty in assessing precisely the viability of the 2 ruptured urethral ends. Secondary stricture, incontinence, and impotence rates are also high[12]. Traditionally delayed open urethroplasty, as described by Waterhouse and others, was considered the gold standard repair. The approach was either perineal or transpubic, often requiring staged procedures and associated with extensive blood loss, long operative time, and extended length of hospital stay.

Recently, several authors have reported encouraging results with endoscopic realignment as primary therapy. This procedure requires immediate suprapubic diversion followed by delayed or early endoscopic realignment and is associated with decreased intraoperative blood loss, shortened operative time, decreased length of hospital stay, and less incontinence than open urethroplasty[7].

In the conventional classical approach i.e. early suprapubic cystostomy with delayed urethral reconstruction, virtually $100 \%$ of patients treated with a suprapubic tube (catheter) and delayed reconstruction require urethroplasty, while early realignment obviate the need for about half of these operations. These decrease in the need for surgery has a large positive impact because perineal approach anastomotic urethroplasty can be lengthy and arduous for the surgeon and patient.

On the other hand, series that advocate primary realignment, show a lower rate of urethroplasty as well as the formation of potentially less significant strictures than those treated by primary cystostomy alone. They may be more amenable to endoscopic core through treatment, which often fail in the longer strictures seen with delayed therapy. They also believe that subsequent open repair, if needed, is simpler than that in the delayed urethroplasty[8].

The present work has been carried out to compare the outcome between early primary endoscopic realignment versus suprapubic cystostomy with delayed reconstruction in the management of posterior urethral injury. A total of 60 patients were included, 28 patients underwent early primary endoscopic realignment i.e. Group-A and 32 patients underwent primary suprapubic cystostomy with delayed reconstruction i.e. Group-B. The follow up periods ranged from 3 months to 9 months.

In this study, half of the patients in Group-A was less than 30 years old, $32.1 \%$ between $30-40$ years and rest $17.9 \% 40$ or more than 40 years old. In Group-B, 56.3\% patients was less than 30 years, $34.4 \%$ between $30-40$ years and $9.4 \% 40$ years or more than 40 years. The mean age of patients was almost similar between the groups ( $28.8 \pm 8.4$ vs. $27.4 \pm 7.2$ years, $p=0.486)$. The age of the patients corresponds with the study of Kulkarni et al. and Tazi et al. 
Urethral injury was suspected in those with the history of blunt pelvic trauma together with inability to pass urine, blood at the external urethral meatus, perineal haematoma, suprapubic fullness, high riding prostate and pelvic fracture. The presentations were almost similar in both groups. These are the classical features of urethral injury[1].

In this study, all of the patients of Group-A underwent early primary endoscopic realignment over a catheter. So, there was no question of primary stricture development. But, in case of Group-B, all the patients were left for at least 3 months after initial suprapubic urinary diversion. Therefore, $100 \%$ patients developed strictures which were diagnosed by RGU and MCU before delayed urethral reconstruction. Among them, 7(21.9\%) patients developed simple and long segmented stricture and 25(78.1\%) patients exhibited complex stricture. Koraitim and Mouraviev et al. conducted two studies and they got $97 \%$ and $100 \%$ strictures respectively.

In case of Group-A, after early primary endoscopic realignment, all the patients were counseled for regular follow up and in case of Group-B, follow up programme was conducted after delayed realignment by anastomotic urethroplasty. In both groups the total follow up periods ranged from 3 months to 9 months and was done at removal of catheter, at $3^{\text {rd }}$ month, at $6^{\text {th }}$ month and at $9^{\text {th }}$ month after procedure to search 3 potential complications like incontinence, impotence and secondary stricture.

In case of Group-A, all of the patients had minimum blood loss during endoscopic realignment, while majority (81.3\%) of patients in Group-B had a history of maximum blood loss during delayed open urethral reconstruction. Similarly, the mean duration of operation was found significantly higher in Group-B than in Group-A (2.5 \pm 0.4 vs. $1.2 \pm 0.4 \mathrm{hrs}$. $p<0.001$ ). The mean hospital stay was two times higher in Group-B compared to Group-A (6.9 \pm 1.8 vs. $3 \pm 0.1$ days, $p<0.001)$. In $7(21.9 \%)$ cases, anastomotic urethroplasty was associated with partial or total pubectomy.

Incontinence is one of the complications of posterior urethral injury either during injury or after the corrective procedure. Non of the patients in Group-A developed incontinence throughout the observation periods (from removal catheter to 9 months) which corresponds with the study of Guille et al, Moudouni et al. and Tuzi et al. But in case of Group-B, it was $15.6 \%$ at removal of catheter and at month 3 . In further follow up visit, it was $18.8 \%$. Mouraviev et al. stated a comparative experience of early endoscopic realignment versus delayed urethroplasty in the treatment of posterior urethral disruption associated with pelvic fracture. They found out $24.9 \%$ incontinence rate in delayed urethroplasty. Cooperberg et al. conducted another study on delayed urethroplasty and they also stated $13 \%$ incontinence rate.

Impotence is a burning issue in the patients with posterior urethral distraction defect both before and after treatment. In present study, postoperative impotence was significantly less in Group-A than that in Group-B throughout the period of observation (14.3\% vs. $37.5 \%$, $\mathrm{p}=0.042)$. Rehman et al, Jepson et al. and Tazi et al. stated $40 \%, 37.5 \%$ and $19.4 \%$ impotence respectively in case of early primary endoscopic realignment of posterior urethral distraction defect (PUDD). In case of delayed urethroplasty, the rate of impotence corresponds with the study conducted by Mouraviev et al. which was $42.1 \%$.

Postoperative recurrent stricture formation is another issue in the management of PUDD. In this study, at removal of urethral catheter, stricture formation was observed to be similar in Group-A and Group-B (14.3\% vs. $21.9 \%, p=0.448)$. At month 3 , stricture formation was significantly higher in Group-A than that in GroupB (42.9\% vs. $15.6 \%, p=0.020)$. At month 6 , about $18 \%$ of patients in Group-A had stricture, but none of patients in Group-B was found so $(p=0.018)$. There are so many international publications on this ground, where recurrent stricture formation after early primary endoscopic alignment was higher, e.g. Guille et al. (1991), Rehman et al, Jepson et al. and Moudouni stated $60 \%, 54 \%, 50 \%$ and $41 \%$ respectively. On the other hand, low rate of stricture formation in case of delayed reconstruction was described by Cooperberg et al. which was $14 \%$.

All of the recurrent strictures in both groups were treated by simple OIU, but all of the delayed reconstruction in case of Group-B were performed by anastomotic urethroplasty with or without partial or total pubectomy. Overall results between two groups were compared. After the study, we got some remarkable information. With 
the advent of endourology, early primary endoscopic realignment in the management of PUDD is good option. It is simple, less time consuming and associated with good outcome and less postoperative complications except recurrent stricture formation which is higher than that of delayed reconstruction group. The recurrent strictures are amenable to be corrected by simple OIU. With meticulous approach by expert surgeon, the rate of recurrent stricture formation can be reduced.On the other hand, primary cystostomy with delayed reconstruction by anastomotic urethroplasty is a laborious, time consuming and difficult procedure which is invariably associated with more per-operative blood loss and postoperative complications like incontinence, impotence etc.

\section{Conclusions:}

Early primary endoscopic realignment of traumatic posterior urethral disruption is a simple, less traumatic, safe, and rapid technique. It provides a low morbidity and less postoperative complications. Though recurrent stricture formation is higher but the strictures are simple and short and amenable to be corrected endoscopically. It may be considered as initial therapy in the management of posterior urethral distraction defect over suprapubic cystostomy with delayed reconstruction. Preliminary results with early primary endoscopic realignment in the management of posterior urethral distraction defect are encouraging. A long-term followup is necessary to observe the possible advantages of this new technique against the standard suprapubic cystostomy with delayed reconstruction.

\section{Acknowledgements}

We highly grateful Prof. SM Mahbubul Alam for his kind help in preparing their manuscript.

\section{Conflict of Interest : None Declared}

\section{References:}

1. McAninch, JW 2008, 'Injuries to the Genitourinary Tract', in Smith's General Urology, $17^{\text {th }}$ edn, eds EA Tanagho and JW McAninch, The McGrow-Hill Companies, USA, 278-296.

McAninch, J and Morey, A 2009, 'Reconstruction of Posterior Urethral Distraction Injuries: Outcome Analysis in 82 Patients', The Journal of Urology, 157, 2, 506-510.

2. Tazi, H, Ouali, M, Said Moudouni, MHFID LRHORFI, Tazi, K and Lakrissa, A 2003, 'The endoscopic realignment in the post-traumatic rupture of the posterior urethra', Prog Urol, 13, 6, 1345-1350.

3. Morey, AF and Rozanski, TA 2007, 'Genital and Lower Urinary Tract Trauma', in Campbell-Walsh UROLOGY, $9^{\text {th }}$ edn, eds LR Kavoussi, AC Novick, LW Partin, CA Peters and AJ Wein, Saunders Elsevier, 1600 Jhon F. Kennedy Blvd, Ste 1800, Philadelphia, PA 19103-2899, 2649-2662.

4. Fan, EW, Cheng, TC, Lin, $\mathrm{H}$ and Chiu, AW 2000, 'Early Primary Endoscopic Realignment for Posterior Urethral Injury in a Patient with Malgaigne's Fracture: Case Report', J UROL ROC, 11, 2, 86-89.

5. Porter, JR, Takayama, TK and Defalco, AJ 1997, 'Traumatic posterior urethral injury and early realignment using magnetic urethral catheters', The Journal of Urology, 158, 425-430.

6. Koraitim, MM, Atta, MA, Fattah, GA and Ismail HR 2003, 'Mechanism of continence after repair of posttraumatic posterior urethral strictures', Adult Urology, 61, 287-290.

Koraitim, M 2009, 'Pelvic fracture urethral injuries: the unresolved controversy', The Journal of Urology, 161, 5, 1433-1441.

7. Rehman, J, Samadi, D, Riccardi, R and Kreutzer, E 1998, 'Early Endoscopic Realignment as Primary Therapy for Complete Posterior Urethral Disruptions', Journal of Endourology12, 3.

8. Mouraviev, VB, Coburn, M and Santucci RA 2005, 'The treatment of posterior urethral distraction associated with pelvic fractures: comparative experience of early realignment versus delayed urethroplasty', The Journal of Urology, 173, 3, 873-876.

9. Hadjizacharia, P, Inaba, K, Teixeira, PGR, Kokorowski, P, Demetriades, D and Best, C 2008, 'Evaluation of Immediate Endoscopic Realignment as a treatment modality for traumatic urethral injuries,' The Journal of TRAUMA,64, 6.

10. Shrinivas, RP and Dubey, D 2010, 'Primary urethral realignment should be the preferred option for the initial management of posterior urethral injuries, 'Indian J. Urol, 26, 2, 310-313.

11. Koraitim, MM 1996, 'Pelvic fracture urethral injuries: evaluation of various methods of management' , The Journal of Urology, 156, 1288-1291. 
12. Guille, F, Cipolla, B, Leveque, JM, Guirassy, S, Olivo, JF and Lobel, B 1991, 'Early Endoscopic Realignment of Complete Traumatic Rupture of the Posterior Urethra' , British Journal of Urology, 68, 178-180.

13. Podesta, ML, Medel, R, Castera, R and Ruarte, A 1997, 'Immediate management of posterior urethral disruptions due to pelvic fracture: therapeutic alternatives', The Journal of Urology, 157, 14441448.

14. Kulkarni, SB, Barbagli, G, Kulkarni, JS, Romano, G and Lazzeri, M 2010, 'Posterior Urethral Stricture After Pelvic Fracture Urethral Distraction Defects in Developing and Developed Countries, and Choice of Surgical Technique', The Journal of Urology, 183, 1049-1054.
15. Moudouni, SM, Patard, JJ, Manunta, A, Guiraud, P, Lobel, B and Guille, F 2001, 'Early endoscopic realignment of post-traumatic posterior urethral disruption' , Adult Urology, 57, 628-632.

16. Jepson, BR, Boullier, JA, Moore, RG and Parra, RO 1999, 'Traumatic posterior urethral injury and early primary endoscopic realignment: evaluation of long-term follow-up', Adult Urology, 53, 12051210.

\author{
Abbreviations: \\ IER : Immediate Endoscopic Realignment \\ PUDD : Posterior Urethral Destruction Defect
}

